\title{
X-ray burst studies with the JENSA gas jet target
}

Konrad Schmidt ${ }^{1,2, \star}$, Kelly A. Chipps ${ }^{3}$, Sunghoon Ahn ${ }^{1,2}$, Jacob M. Allen ${ }^{4}$, Sara Ayoub ${ }^{1,2,5}$, Daniel W. Bardayan ${ }^{4}$, Jeffrey C. Blackmon ${ }^{6}$, Drew Blankstein ${ }^{4}$, Justin Browne ${ }^{1,2,5}$, Soomi Cha ${ }^{7}$, Kyung Yuk Chae ${ }^{7}$, Jolie Cizewski ${ }^{8}$, Catherine M. Deibel ${ }^{6}$, Eric Deleeuw ${ }^{1,2,5}$, Orlando Gomez ${ }^{9}$, Uwe Greife $^{10}$, Ulrike Hager ${ }^{1,5}$, Matthew R. Hall ${ }^{4}$, Katherine L. Jones ${ }^{11}$, Antonios Kontos ${ }^{12}$, Raymond L. Kozub ${ }^{13}$, Eunji Lee ${ }^{7}$, Alex Lepailleur ${ }^{8}$, Laura E. Linhardt ${ }^{1}$, Milan Matos ${ }^{14}$, Zach Meisel ${ }^{2,15}$, Fernando Montes ${ }^{1,2}$, Patrick D. O'Malley ${ }^{4}$, Wei Jia Ong ${ }^{1,2,5}$, Steven D. Pain ${ }^{3}$, Alison Sachs ${ }^{11}$, Hendrik Schatz ${ }^{1,2,5}$, Kyle T. Schmitt ${ }^{11}$, Karl Smith ${ }^{11}$, Michael S. Smith ${ }^{3}$, Natã F. Soares de Bem ${ }^{13,16}$, Paul J. Thompson ${ }^{11}$, Rebecca Toomey ${ }^{8,17}$, and David Walter ${ }^{8}$

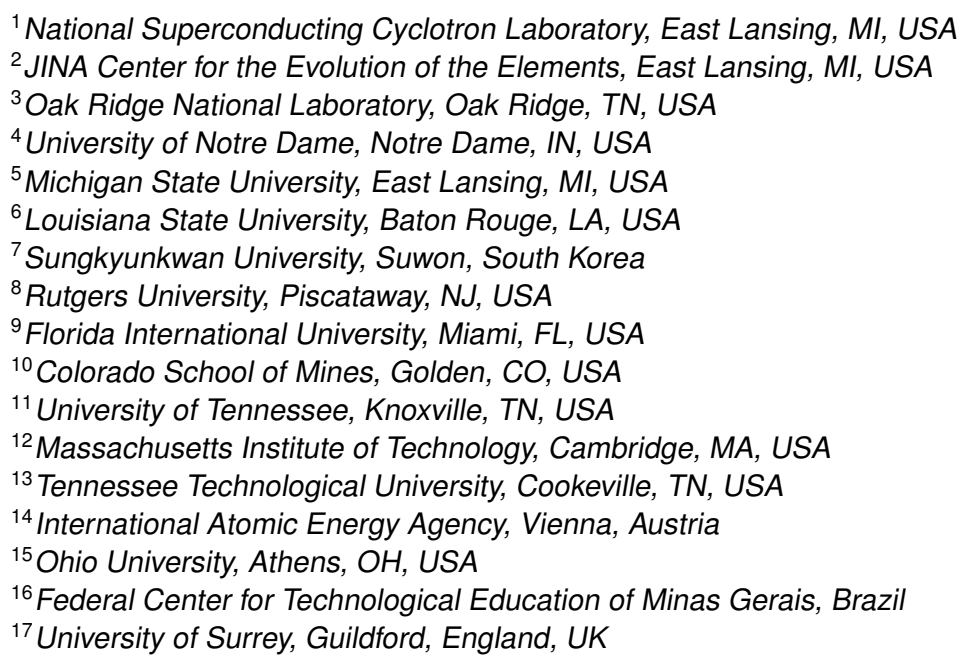

\begin{abstract}
When a neutron star accretes hydrogen and helium from the outer layers of its companion star, thermonuclear burning enables the $\alpha$ p-process as a break out mechanism from the hot $\mathrm{CNO}$ cycle. Model calculations predict $(\alpha, \mathrm{p})$ reaction rates significantly affect both the light curves and elemental abundances in the burst ashes. The Jet Experiments in Nuclear Structure and Astrophysics (JENSA) gas jet target enables the direct measurement of previously inaccessible $(\alpha, \mathrm{p})$ reactions with radioactive beams provided by the rare isotope re-accelerator ReA3 at the National Superconducting Cyclotron Laboratory (NSCL), USA. JENSA is going to be the main target for the Recoil Separator for Capture Reactions (SECAR) at the Facility for Rare Isotope Beams (FRIB). Commissioning of JENSA and first experiments at Oak Ridge National Laboratory (ORNL) showed a highly localized, pure gas target with a density of $\sim 10^{19}$ atoms per square centimeter. Preliminary results are presented from the first direct cross section measurement of the ${ }^{34} \operatorname{Ar}(\alpha, \mathrm{p}){ }^{37} \mathrm{~K}$ reaction at NSCL.
\end{abstract}

^e-mail: schmidtk@nscl.msu.edu 


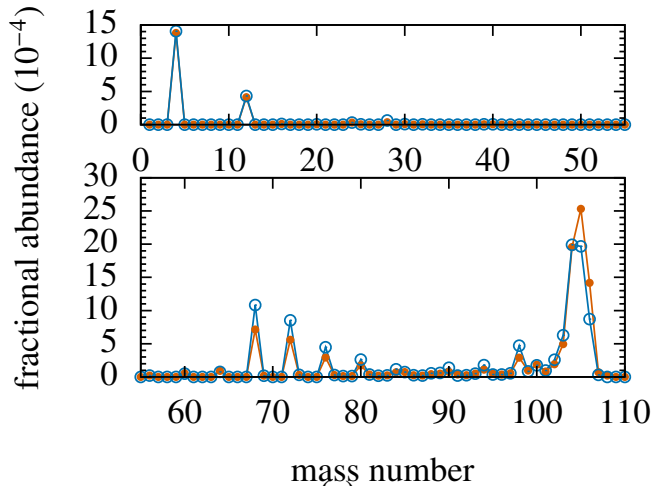

(a)

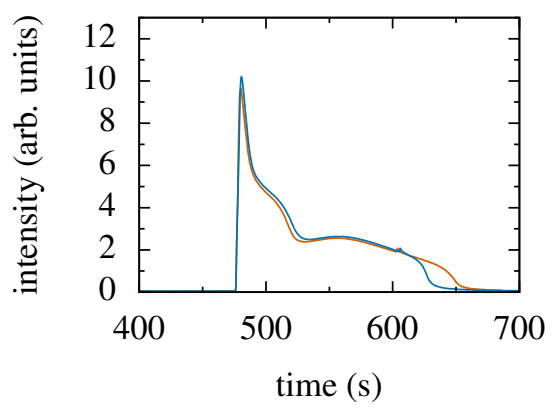

(b)

Figure 1: Final abundance plot (a) and light curves (b) from a single-zone X-ray burst calculation. The nominal rate of the ${ }^{34} \operatorname{Ar}(\alpha, \mathrm{p})^{37} \mathrm{~K}$ reaction has been decreased (red) and increased (blue) by a factor of 100 .

\section{Introduction}

A type I X-ray burst occurs when a neutron star accretes material from the outer layers of its binary companion. The transfered helium and hydrogen accumulates on the neutron star surface resulting eventually in a thermonuclear explosion, and triggering reaction chains from the triple- $\alpha$ reaction via the hot CNO cycle, breaking out in the $\alpha$ p-process, and leading into the rp-process $[1,2]$. Sensitivity studies [3-5] show the $\alpha$ p-process reaction flow passing through the ${ }^{34} \operatorname{Ar}(\alpha, \mathrm{p})^{37} \mathrm{~K}$ reaction. Only little is known experimentally about the reaction cross section. Theoretical calculations based on the Hauser-Feshbach statistical approach deviate from a recent indirect study [6] by a factor of about 100 at astrophysical energies.

In order to study the influence of the ${ }^{34} \operatorname{Ar}(\alpha, \mathrm{p})^{37} \mathrm{~K}$ reaction, its nominal rate has been decreased and increased by a factor 100 in a single-zone X-ray burst calculation with an initial hydrogen abundance of 0.5 . The reaction shows a broad effect on multiple species in the burst ashes (Figure 1a), as a higher rate increases the hydrogen to seed ratio of the subsequent rp-process. Furthermore, changing only that one reaction rate directly affects the burst light curve (Figure 1b). An increased reaction rate accelerates the reaction flow and the burst burns out faster.

In order to investigate discrepancies between theoretical calculations and indirect measurements, a direct study of the ${ }^{34} \operatorname{Ar}(\alpha, \mathrm{p}){ }^{37} \mathrm{~K}$ reaction in inverse kinematics has been performed at the National Superconducting Cyclotron Laboratory (NSCL). Since current rare isotope beam intensities are not sufficient to measure at astrophysically relevant energies, the reaction has been studied above the Gamow window, at $E_{\mathrm{CM}}=5.8$ and $6.1 \mathrm{MeV}$ in the center of mass frame.

\section{Experimental Setup}

The rare isotope beam facility ReA3 [7] at NSCL on the campus of Michigan State University, USA, provided a radioactive ${ }^{34} \mathrm{Ar}$ beam with an average intensity of $\sim 3000$ particles per second (pps). A chemically pure, highly-localized helium target with high density and low energy straggling was 
generated by the Jet Experiments for Nuclear Structure and Astrophysics (JENSA) gas jet system. The recirculating gas system created a jet of $\sim 10^{19}$ atoms $/ \mathrm{cm}^{2}$ [8] by compressing the gas to about 30 atmospheres and forcing it through a nozzle with a minimum inner diameter of $1 \mathrm{~mm}$. This high pressure gas constituted the main target area where the $(\alpha, \mathrm{p})$ reaction took place. The jet streamed into a receiver system and the gas was pumped through multiple pumping stages leading back to the compressor. The vanishingly small fraction of the gas from the jet that streamed into the vacuum chamber was evacuated by a magnetically levitated turbo molecular pump. Further upstream along the beam line, four additional turbo molecular pumps reduced the pressure down to $10^{-7} \mathrm{mbar}$.

The jet was surrounded by multiple highly segmented silicon strip detectors, two Super Oak Ridge Rutgers University Barrels (SuperORRUBA) [9] upstream and downstream of the jet, as well as the Silicon Detector Array (SIDAR) [10] at forward angles. Upstream SuperORRUBA is composed of six single Micron style BB15 silicon detectors of 1-mm thickness. Downstream, four of the six BB15 detectors were stacked each with a Micron style BB10 silicon detector of $65-\mu \mathrm{m}$ creating $\Delta E-E$ telescopes. SIDAR is arranged by five $65-\mu \mathrm{m}$ and five $1-\mathrm{mm}$ thick Micron style YY1 detectors as telescopes in a lampshade configuration.

A position sensitive ionization chamber (PSIC) [11] was installed downstream of the vacuum chamber, separated by a $3-\mu \mathrm{m}$ thick Mylar $\left(\mathrm{H}_{8} \mathrm{C}_{10} \mathrm{O}_{4}\right)$ window, to measure beam current and beam composition, as well as reaction recoils. The PSIC was divided in four sections in order to measure the beam and recoil position in $\mathrm{X}$ (section 1) and $\mathrm{Y}$ (section 2) relative to the beam axis, as well as the energy loss in those first two sections, the intermediate energy loss $\Delta E$ (section 3), and the residual energy $E_{\text {res }}$ (section 4). The PSIC was filled with isobutane $\left(\mathrm{C}_{4} \mathrm{H}_{10}\right)$ at a pressure of about $20 \mathrm{mbar}$ chosen to completely stop the beam inside the chamber.

Preamplifier signals from the silicon detectors were passed to the Application Specific Integrated Circuits (ASICs) data acquisition [12, 13] and combined with the Digital Data Acquisition System (DDAS) [14] output from the preamplified PSIC chamber signals, including 32 channels from each of the $\mathrm{X}$ - and Y-section, one channel from $\Delta E$ and one from $E$. Signals from both systems were then recorded with a synchronized time stamp.

Beam contaminants were exclusively caused by ${ }^{34} \mathrm{Ar}$ radioactive decay upstream of the JENSA target. The ${ }^{34} \mathrm{Ar}(\sim 60 \%)$ rare-isotope beam was contaminated with the daughter ${ }^{34} \mathrm{Cl}(\sim 30 \%)$ and the grand daughter ${ }^{34} \mathrm{~S}(\sim 10 \%)$. Recoils from the $(\alpha, \mathrm{p})$ reactions with ${ }^{34} \mathrm{Ar}$ and with beam contaminants will be disentangled by the different energy losses in each of the four sections in the PSIC.

\section{Preliminary Results}

Signals in the silicon detector telescope in coincidence with recoil signals from the PSIC are shown in Figure 2a. Particle identification in this $\Delta E-E$ spectrum clearly separates scattered $\alpha$ particles and protons from the $(\alpha, \mathrm{p})$ reaction. Note that the acceptance angle of the PSIC is optimized for proton recoils but recoils from $\alpha$ scattering are mostly cut off by the size of the PSIC entrance window. As a consequence, only $\alpha$ particles in coincidence with scattered recoils at small laboratory angles with respect to the beam axis are shown in Figure 2a.

The gated proton signals from Figure 2a follow very well the expected kinematic lines (Figure 2b). Since protons are emitted from decays into various excited states of ${ }^{37} \mathrm{~K}$ and ${ }^{37} \mathrm{Ar}$, as well as into the ground and the first excited state of ${ }^{37} \mathrm{Cl}$, the measured proton signals in the silicon detector telescopes cover a wide energy spread (Figure $2 \mathrm{~b}$ ) instead of following a single kinematic curve. Data analysis including signals from the eight single silicon detectors (two downstream and six upstream of the jet position) is underway. The ${ }^{34} \operatorname{Ar}(\alpha, \mathrm{p}){ }^{37} \mathrm{~K}$ reaction cross section will be determined from the wellknown target thickness [8], detected protons and the effective beam current. 


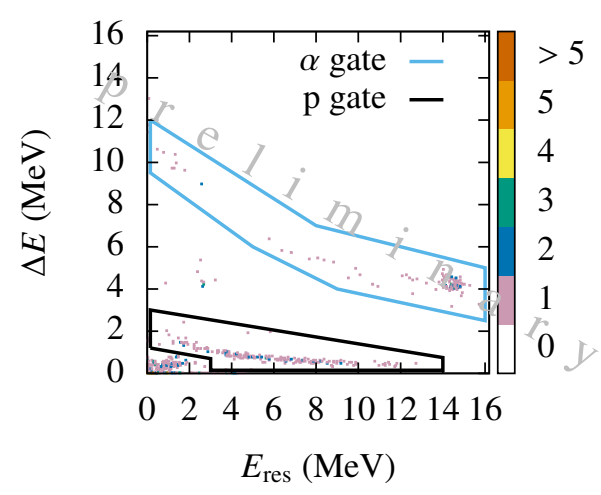

(a)

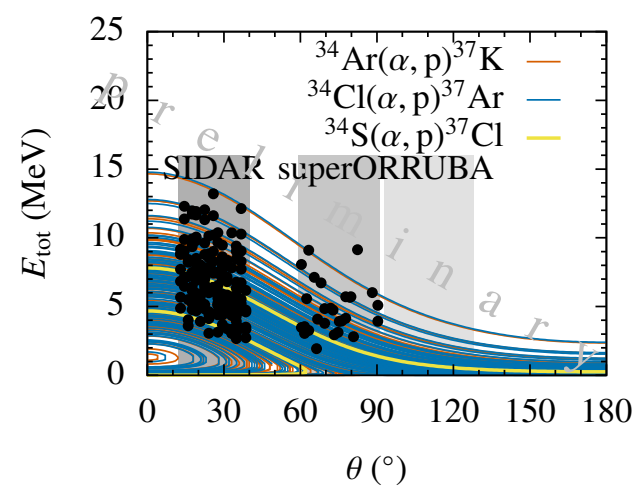

(b)

Figure 2: (a) Preliminary $\alpha$ and proton signals in coincidence with recoil signals in the PSIC. The PSIC covers the full recoil angle for $(\alpha, \mathrm{p})$ reactions with ${ }^{34} \mathrm{Ar}$ and its beam contaminations. Recoils from $\alpha$ scattering are only partly covered by the PSIC. (b) Protons from (a) and kinematic lines. In downstream SuperORRUBA (59 ${ }^{\circ}$ to $91^{\circ}$ ) only four detectors are arranged as telescopes and contribute to the $\Delta E-E$ gate, upstream superORRUBA exclusively comprises single layer detectors. Reactions on ${ }^{34} \mathrm{~S}$ emit protons from transitions only to the ground and the first excited state whereas protons from reactions with ${ }^{34} \mathrm{Ar}$ and ${ }^{34} \mathrm{Cl}$ contribute to several excited states.

\section{Acknowledgements}

This research is supported by the National Science Foundation in part under Grant No. PHY-1430152 (JINA Center for the Evolution of the Elements), Grant No. PHY-1419765, Grant No. PHY-1404218, by the U.S. Department of Energy (DoE), Office of Nuclear Physics and National Nuclear Security Administration, and by the Laboratory Directed Research and Development Program of Oak Ridge National Laboratory, managed by UT-Battelle, LLC, for DoE.

\section{References}

[1] R. K. Wallace and S. E. Woosley, Astrophys. J. Suppl. Ser.45, 389 (1981)

[2] H. Schatz et al. Phys. Rep. 294, 167 (1998)

[3] J. L. Fisker, H. Schatz, and F.-K. Thielemann, Astrophys. J. Suppl. Ser.174, 261 (2008)

[4] A. Parikh et al., Astrophys. J. Suppl. Ser.178, 110 (2008)

[5] R. H. Cyburt et al., Astrophys. J. 830, 55 (2016)

[6] A. M. Long et al., Phys. Rev. C 95, 055803 (2017)

[7] D. Leitner et al., Nucl. Instrum. Meth. B 317, 235 (2013)

[8] K.A. Chipps et al., Nucl. Instrum. Meth. A 763, 553 (2014)

[9] D. W. Bardayan et al., Nucl. Instrum. Meth. A 711, 160 (2013)

[10] D. W. Bardayan et al., Phys. Rev. C 63, 065802 (2001)

[11] K. Y. Chae et al., Nucl. Instrum. Meth. A 751, 6 (2014)

[12] M. S. Wallace et al., Nucl. Instrum. Meth. A 573, 418 (2007)

[13] M. S. Wallace et al., Nucl. Instrum. Meth. A 583, 302 (2007)

[14] K. Starosta et al., Nucl. Instrum. Meth. A 610, 700 (2007) 